\begin{tabular}{ll} 
PROS & PROCEEDINGS \\
\hline
\end{tabular}

\title{
The ENUBET monitored neutrino beam: a progress report
}

C. Brizzolari, ${ }^{c, d, *}$ F. Acerbi, ${ }^{a}$ I. Angelis, ${ }^{u}$ M. Bonesini, ${ }^{c}$ F. Bramati, ${ }^{c, d}$ A. Branca, ${ }^{e, j}$ G. Brunetti, ${ }^{e}$ M. Calviani, ${ }^{f}$ S. Capelli, ${ }^{b, c}$ S. Carturan, ${ }^{g}$ M.G. Catanesi, ${ }^{h}$ S. Cecchini, ${ }^{i}$ N. Charitonidis, ${ }^{f}$ F. Cindolo, ${ }^{i}$ G. Collazuol, ${ }^{e} j$ F. Dal Corso, ${ }^{e}$ C. Delogu, ${ }^{e, j}$ G. De Rosa, ${ }^{k}$ A. Falcone,${ }^{c, d}$ B.Goddard, ${ }^{f}$ A. Gola, ${ }^{a}$ F. lacob, ${ }^{e, j}$ C. Jollet, ${ }^{l, n}$ V. Kain, ${ }^{f}$ B. Klicek, ${ }^{t}$ Y. Kudenko, ${ }^{m}$ Ch. Lampoudis, ${ }^{u}$ M. Laveder, ${ }^{e j}$ A. Longhin,,${ }^{e j}$ L. Ludovici, ${ }^{o}$ E. Lutsenko, ${ }^{b, c}$ L. Magaletti, ${ }^{h}$ G. Mandrioli, ${ }^{i}$ A. Margotti, ${ }^{i}$ V. Mascagna,,${ }^{b, c}$ N. Mauri, ${ }^{i}$ L. Meazza, ${ }^{c, d}$ A. Meregaglia, ${ }^{n}$ M. Mezzetto, ${ }^{e}$ M. Nessi, ${ }^{f}$ A. Paoloni, ${ }^{q}$ M. Pari, ${ }^{e}, j$ E.G. Parozzi, ${ }^{c, d}$ L. Pasqualini, ${ }^{i, r}$ G. Paternoster ${ }^{a}$ L. Patrizii, ${ }^{i}$ M. Pozzato, ${ }^{i}$ M. Prest,${ }^{b, c}$ F. Pupilli, ${ }^{e}$ E. Radicioni, ${ }^{h}$ C. Riccio, ${ }^{k, s}$ A.C. Ruggeri, ${ }^{k}$ D. Samponidis, ${ }^{u}$ C. Scian, ${ }^{j}$ G. Sirri, ${ }^{i}$ M. Stipcevic, ${ }^{t}$ M. Tenti, ${ }^{i}$ F. Terranova,,${ }^{c, d}$ M. Torti, ${ }^{c, d}$ S.E. Tzamarias, ${ }^{i}$ E. Vallazza, ${ }^{c}$ F. Velotti ${ }^{f}$ and L. Votano ${ }^{q}$

${ }^{a}$ Fondazione Bruno Kessler (FBK) and INFN TIFPA, Trento, Italy

${ }^{b}$ DiSAT, Università degli studi dell'Insubria, via Valleggio 11, Como, Italy

${ }^{c}$ INFN, Sezione di Milano-Bicocca, piazza della Scienza 3, Milano, Italy

${ }^{d}$ Phys. Dep. Università degli Studi di Milano-Bicocca, piazza della Scienza 3, Milano, Italy

e INFN Sezione di Padova, via Marzolo 8, Padova, Italy

${ }^{f}$ CERN, Geneva, Switzerland

${ }^{g}$ INFN Laboratori Nazionali di Legnaro, Viale dell'Università, 2 - Legnaro (PD), Italy

${ }^{h}$ INFN Sezione di Bari, via Amendola 173, Bari, Italy

${ }^{i}$ INFN, Sezione di Bologna, viale Berti-Pichat 6/2, Bologna, Italy

${ }^{j}$ Phys. Dep. Università di Padova, via Marzolo 8, Padova, Italy

${ }^{k}$ INFN, Sezione di Napoli, via Cinthia, 80126, Napoli, Italy

${ }^{l} I P H C$, Université de Strasbourg, CNRS/IN2P3, Strasbourg, France

${ }^{m}$ Institute of Nuclear Research of the Russian Academy of Science, Moscow, Russia

${ }^{n}$ Centre de Etudes Nucleaires de Bordeaux Gradignan, 19 Chemin du Solarium, Bordeaux, France

${ }^{o}$ INFN, Sezione di Roma 1, piazzale A. Moro 2, Rome, Italy

${ }^{p}$ CENBG, Université de Bordeaux, CNRS/IN2P3, 33175 Gradignan, France

${ }^{q}$ INFN, Laboratori Nazionali di Frascati, via Fermi 40, Frascati (Rome), Italy

${ }^{r}$ Phys. Dep. Università di Bologna, viale Berti-Pichat 6/2, Bologna, Italy

${ }^{s}$ Phys. Dep. Università degli Studi di Napoli Federico II, via Cinthia, 80126, Napoli, Italy

${ }^{t}$ Center of Excellence for Advanced Materials and Sensing Devices, Ruder Boskovic Institute, HR-10000 Zagreb, KR

${ }^{u}$ Aristotle University of Thessaloniki, Thessaloniki 541 24, Greece

E-mail: claudia.brizzolari@mib.infn.it

${ }^{*}$ Speaker 
The ENUBET experiment, included in the CERN Neutrino Platform effort as NP06/ENUBET, is developing a monitored neutrino beam based on conventional techniques. The aim is to constrain the flux and the flavor composition with a precision of $\mathrm{O}(1 \%)$ by monitoring the associated charged leptons produced in the decay tunnel. The narrow momentum width $(<10 \%)$ of the beam also allows to obtain a precise measurement of the neutrino energy. This paper summarizes the latest development and the status of the project.

*** The European Physical Society Conference on High Energy Physics (EPS-HEP2021), ***

*** 26-30 July $2021 * * *$

*** Online conference, jointly organized by Universität Hamburg and the research center DESY *** 


\section{The idea of monitored neutrino beams}

ENUBET (Enhanced NeUtrino BEams from kaon Tagging) aims to develop a narrow-band, monitored neutrino beam for the precision era of $v$ physics [1]. The ENUBET ERC project started in 2016 and, due to the COVID19 pandemic, it was extended up to November 2022. In 2019 ENUBET was also approved by CERN as a Neutrino Platform experiment, NP06 [2], [3]. The physics goal of NP06/ENUBET is to constrain the uncertainties of the flux and hence on the cross sections at the GeV scale of $v_{e}$ and $v_{\mu}$ at the order of $1 \%$, the energy of the neutrino at $10 \%$ and the flavor composition at 1\%. Lowering the cross sections systematic budged under $3 \%$ is essential for the DUNE and HyperKamiokande discovery reach. These goals are achieved by the instrumented decay tunnel (tagger), which allows the monitoring of the positrons from the $K_{e 3}$ decay. The $e^{+}$ counting then translates to a direct $v_{e}$ flux determination. $v_{\mu}$ flux determination is obtained by extending the monitoring to the muons from $K \rightarrow \mu v_{\mu}$ and $\pi \rightarrow \mu v_{\mu}$. Direct measurements of the fluxes inside the decay tunnel allow to avoid uncertainties from Protons-on-Target (POT), hadron-production, and beam line efficiency.

\section{Beamline design}

The ENUBET transfer line is optimized for a $8.5 \mathrm{GeV} / \mathrm{c}$ reference particle and the two latest design for a static transfer line are referred to as TLR5 and TLR6. Both these transfer lines consider two normal-conduction bending dipoles with $1.8 \mathrm{~T}$ field that provide a bending angle of $14.8^{\circ}$ w.r.t. the primary proton line. The aperture radius of the quadrupoles is the same for the two configurations $(15 \mathrm{~cm})$, but their number and position differ from one design to the other. This is because TLR5 is optimized for a 5\% momentum bite, while the TLR6 was optimized considering a $10 \%$ momentum bite and a new improved target made of graphite, to maximise the yields of mesons in the region of interest. The optimization was carried out with FLUKA and G4beamline. Thanks to the TLR6 design, the $v_{e}$ from the target region at low-E are significantly suppressed and the estimated event rate on a $500 \mathrm{t}$ detector installed $100 \mathrm{~m}$ downstream the target is $10^{4} v_{e}^{C C}$ for $4.5 \times 10^{19} \mathrm{POT} / \mathrm{y}$.

\section{Monitoring instrumentation}

The monitoring of positrons from the $K_{e 3}$ decay and $e^{+} / \pi^{ \pm} / \mu$ separation are performed by a longitudinally segmented calorimeter that surrounds the axis of the decay tunnel. Its energy resolution must be $<25 \% / \sqrt{\mathrm{GeV} / E}$ to complement the longitudinal showering for $e / \pi$ separation. The technology of choice is that of the sampling calorimeter with lateral readout, in which the $0.5 \mathrm{~cm}$ thick plastic scintillator tiles have a groove on each side for the WLS fiber that transports the scintillator light to the SiPM, placed outside of the decay tunnel. The plastic scintillator tiles are interleaved with $1.5 \mathrm{~cm}$ thick iron tiles as absorbing medium. The building block of the positron tagger is the LCM (Lateral Compact Module) made by 5 plastic scintillator tiles and 5 iron tiles. The LCM dimensions are $3 \times 3 \times 10 \mathrm{~cm}^{3}$, covering $\sim 4.3 X_{0}$. The ten (two per scintillator tile) WLS fibers are coupled with a single $4 \times 4 \mathrm{~mm}^{2} \mathrm{SiPM}$. The full tagger will be made of 3 radial layers of LCMs. 
A 84 LCMs calorimeter prototype $(3 \times 4 \times 7 \mathrm{LCMs})$ was tested at CERN PS-T9 beamline in September 2018 with a mixed beam of $e^{-}, \mu^{-}$and $\pi^{-}$, with momentum from 1 to $5 \mathrm{GeV} / \mathrm{c}$. In order to simulate the emission angle of the $K_{e 3}$ positrons, the response to electromagnetic showers was tested by tilting the prototype axis of $0,50,100$ and $200 \mathrm{mrad}$ w.r.t. the beam axis [4]. The energy resolution for this prototype was fitted with $\sigma_{E} / E=\sqrt{S / E(\mathrm{GeV}) \oplus C}$, with $S$ stochastic term and $C$ constant term, giving $\sigma_{E} / E \sim 17 \%$ at $1 \mathrm{GeV}$ at $0 \mathrm{mrad}$, well within the requirements.

The rejection of the photons from $\pi^{0}$ decay is performed by the photon veto $\left(t_{0}\right.$-layer $)$, made of plastic scintillator tiles $\left(3 \times 3 \times 0.5 \mathrm{~cm}^{3}\right)$ doublets placed underneath the LCMs every $7 \mathrm{~cm}$, forming inner rings. The $t_{0}$-layer serves also to give precise timing of the particles inside the decay tunnel. The requirements for the $t_{0}$-layer are a photon identification efficiency of $99 \%$, and a time resolution of $\sim 1 \mathrm{~ns}$. Data taking at CERN gave an estimated time resolution of $\sim 400 \mathrm{ps}$. The $t_{0}$-layer capability to separate one MIP from two MIPs was also tested: the signal (1 MIP) selection efficiency for a single $t_{0}$ tile is $87 \%$, while the background (2 MIP-like converted photons) rejection is $89 \%$. This corresponds to a purity of $95 \%$.

\subsection{Demonstrator}

The final ENUBET demonstrator will be tested by 2022 at CERN. It is $1.65 \mathrm{~m}$ long in the longitudinal direction and it spans $90^{\circ}$ in azimuth. All the scintillator tiles are trapezoidal in shape to minimize dead regions and produced with injection molding by UNIPLAST (Moskow) in collaboration with the INR group. The grooves for the WLS fiber are frontal instead of lateral, allowing a safer production and more uniform light collection. A smaller prototype made of 3 LCMs (ENUBINO) has been assembled and is being characterized with cosmic ray tracks at the INFN-LNL laboratories.

\section{Simulation of the facility}

The full ENUBET facility has been simulated with G4Beamline for the beam components, FLUKA for the fine tuning of collimators and shielding and for doses assessment, and GEANT4 for the evaluation of systematics.

The signal from $K_{e 3}$ positrons is identified with a multivariate analysis, based on a Multilayer perceptron Neural Network, which exploits a set of variables describing the energy deposition pattern in the tagger from the longitudinal, transverse and radial segmentation of the calorimeter, and the information from the photon veto. The seed has to be at least $28 \mathrm{MeV}$ in the innermost layer of the calorimeter. This analysis provides an efficiency for positron identification of $\sim 22 \%$ and a signal-to-noise ratio of $\sim 2$.

To tag the $\mu$ from $K_{\mu 2}$ and $K_{\mu 3}$ the seed in the inner layer must be compatible with a MIP, with energy $5<\mathrm{E}<15 \mathrm{MeV}$. Muons are monitored with an efficiency $\sim 34 \%$ and $\mathrm{S} / \mathrm{N}$ of $\sim 6$. Muon monitoring employs the same clustering of the energy deposit as the positron monitoring, but also the longitudinal impact point in the decay tunnel to discriminate between signal and background muons. This technique is not suitable for low energy muons from pion decays, as they do not cross the tagger before impinging on the hadron dump. However, the slow extraction limits the rate of these muons to $1-10 \mathrm{MHz} / \mathrm{cm}^{2}$, and these muons can be tagged by instrumenting the hadron dump with fast and radiation hard detectors, providing a complete monitoring of $v_{\mu}$. 


\subsection{Narrow band off-axis technique}

The narrow momentum of the beam $(\mathrm{O}(5-10 \%))$, allows to correlate the neutrino energy with the position of the interaction vertex in a 500 ton LAr detector, with a cross sectional area of $6 \times 6 \mathrm{~m}^{2}$ and placed at short distance ( $90 \mathrm{~m}$ from entrance of decay tunnel, $50 \mathrm{~m}$ from hadron dump). The results of this technique applied to the ENUBET beam, simulated using the realistic beam composition and profile provided by the latest version of the transfer line (TLR6), give a precision on the neutrino energy of $8 \%-25 \%$ in DUNE energy domain and 30\% for HyperKamiokande.

\subsection{Assessment of neutrino flux systematics}

A full systematic assessment is being performed to demonstrate that ENUBET can achieve the $1 \%$ goal on $v_{e}$ and $v_{\mu}$ fluxes by constraining the neutrino flux with the data from the reconstructed leptons; this is being perfomed with a software framework written within RooFit. The systematic uncertainties are reduced by constraining the parameters a posteriori, using all neutrino monitoring data (from the tagger, the hadron dump, etc). These data constrained parameters are then used to reweight the MonteCarlo simulation and get a higher precision on the neutrino flux. This algorithm has already been validated with a toy Monte Carlo. The next step is to build a model based on real hadroproduction data. The tests with the toy MC started with an initial systematic uncertainty on the neutrino spectrum of $\sim 15 \%$, that dropped to $\sim 1.8 \%$ after reweighting. Further improvement are expected by employing the azimuthal and angular distributions of the reconstructed leptons.

\section{References}

[1] A. Longhin, L. Ludovici and F. Terranova, Eur. Phys. J. C 75 (2015) 155

[2] F. Acerbi et al., CERN-SPSC-2018-034, SPSC-I-248, Geneva, 2018

[3] F. Acerbi et al., CERN-SPSC-2021-013, SPSC-SR-290, Geneva, 2021

[4] F. Acerbi et al.2020 JINST 15 P08001 